\title{
Kalman Filter Based Estimation Of Auditory Steady State Response Parameters
}

\author{
Robert Luke and Jan Wouters
}

2015

For submission to IEEE Transactions on Neural Systems and Rehabilitation Engineering

\begin{abstract}
Auditory steady state responses (ASSRs) are brain responses to modulated or repetitive stimuli that can be captured in the EEG recording. ASSRs can be used as an objective measure to clinically determine frequency specific hearing thresholds, to quantify the sensitivity of the auditory system to modulation, and have been related to speech intelligibility. However, the detection of ASSRs is difficult due to the low signal to noise ratio of the responses. Moreover, minimising measurement time is important for clinical applications.

Traditionally ASSRs are analysed using discrete Fourier transform (DFT) based methods. We present a Kalman filter based ASSR analysis procedure and illustrate several benefits over traditional DFT based methods. We show on a data set of 320 measurements that the proposed method reaches valid amplitude estimates significantly faster than the state of the art DFT method.

Further, we provide two extensions to the proposed method. First, we demonstrate information can be incorporated from multiple recording electrodes by extending the system model. Secondly, we extend the model to incorporate artifacts from cochlear implant (CI) stimulation and demonstrate electrically evoked auditory steady state responses (EASSRs) can be accurately measured.
\end{abstract}




\section{Introduction}

Auditory steady state responses (ASSRs) are neural responses to repetitive auditory stimuli [1. These evoked potentials are used clinically to objectively determine frequency specific hearing thresholds [2, 3]. Typical ASSR stimuli are amplitude or frequency modulated carriers. Low frequency amplitude modulated ASSRs can be used to probe the auditory systems sensitivity to modulation rates found in the speech envelope. The presence and strength of ASSRs have been related to speech in noise perception [4, 5].

Hearing thresholds can be determined objectively by decreasing the stimulus intensity until no response is detected [2]. Longer measurements provide more accurate ASSR amplitude estimates. Increasing measurement time at each intensity from 5 to 10 min increases the correlation between objective and behavioural thresholds [3]. In clinical conditions the measurement time should be as short as possible while maintaining a high level of accuracy.

To elicit ASSRs, stimuli are presented to the listener at a set repetition or modulation rate $\left(f_{m}\right)$ using clicks, amplitude or frequency modulated signals. Signals recorded using EEG or MEG can then be found to contain increased energy at $f_{m}$. Measurements also contain sensor noise and spontaneous neural activity, collectively termed measurement noise. Determining accurate estimates of the evoked response amplitude is difficult due to the small signal to noise ratio (SNR). The most commonly used method for determining the amplitude at $f_{m}$ is with a DFT. The gold standard method is to cut the measurement in to smaller segments (epochs), use a DFT to analyse each epoch, and take the mean value of the bin corresponding to $f_{m}$ as the signal amplitude. An estimate of the noise can be achieved by analysing adjacent frequency bins or looking at the variation in the response bin across epochs [6]. The gold standard method to statistically determine the presence of a neural response is to use Hotelling's $\mathrm{T}^{2}$ test [1].

ASSRs can also be elicited with cochlear implant (CI) users [7]. These are termed electrically evoked auditory steady state responses (EASSRs). In the CI population EASSRs have been used to predict thresholds levels [8] and have been related to modulation sensitivity [9]. EEG recordings performed in CI users while their devices are active contain artifacts from the RF transmission and electrical stimulation that obscures the neural response, the artifact contains energy at both the simulation and modulation rates. These artifacts can be removed by interpolating between artifact 
free segments of EEG [8]. This is similar to downsampling, but can not be used with a pre filter as that would smear out the CI artifact.

DFT based analysis of ASSR measurements has several shortcomings. First, the measured response is assumed to be stationary, but effects such as attention or adaptation may cause the ASSR to vary over time [10]. Secondly, the DFT based method can not be applied in real time. Lastly, no consensus has been made on how best to integrate information from multiple electrodes. Various approaches have been proposed to utilise information from multiple recording electrodes including averaging the signals in the time domain [9], averaging the output statistic [11], spatial filtering [12] and blind source separation 13. DFT based EASSR analysis has additional shortcomings. First, the interpolation based artifact removal technique introduces aliasing in the signal. Secondly, only a small portion of the measured data is used in the analysis. Lastly, artifact free segments are required in the data, which are not available when using clinical stimulation parameters.

Here we propose a Kalman filter to estimate the ASSR parameters. This approach requires a model of the signal being measured, we propose a simple sinusoid model with unknown amplitude and phase [14]. When the system model is correct and the noise assumptions of the filter are met, the Kalman filter produces optimal estimates of the system variables. We hypothesise that for low SNR signals the Kalman filter will converge to valid amplitude estimates faster than traditional techniques. We propose two extensions to this Kalman filter technique. To integrate information from multiple recording electrodes we propose a multi input multi output model. And for processing recordings from CI users we propose a system model that incorporates CI artifact information.

The Kalman filter approach provides several advantages over traditional DFT based ASSR processing. First, no assumption of signal stationarity is made. Secondly, changes in the response can be analysed at each sample, opening up new research avenues in to areas such as auditory adaptation or attention. Thirdly, the Kalman filter can be updated in real time during signal acquisition. Lastly, information from multiple recording electrodes can be included in the response estimate. Contrary to DFT based processing, the Kalman filter approach to EASSR processing does not introduce aliasing in the signal and utilises all the measured data.

Accurate estimates of EEG spectral content has many uses. Information about the amplitude at multiple frequencies, or combined with phase estimates, can be used to statistically assess the presence of a neural response. Beyond ASSRs, accurate spectral estimates may be useful in studies 
of attention or neurological disorders [15, 5].

The advantages of a state estimation approach over DFT methods in the analysis of non stationary periodic signals has been previously investigated [16]. A single input single output Kalman filter has been proposed previously for analysis of low SNR EEG signals, specifically the distortion product otoacoustic emissions [17]. But no comparison has been made to current gold standard DFT based methods for measuring ASSRs. Here we compare the proposed method to the current gold standard technique and extend this model to integrate information from multiple electrodes and remove artifacts from EASSRs.

The remainder of this paper is organised as follows. The proposed state space models are defined. The experimental stimuli and recording procedure are described. The statistical tests used to compare the current gold standard DFT procedure and proposed method are described. Results are presented and discussed.

\section{Methods}

In this section the proposed state space models are defined. The stimuli, data collection and signal processing is described. As is the statistical tests to compare results from the current gold standard DFT and proposed methods.

\section{$2.1 \quad$ Models}

Three state space models are introduced to describe: 1) single channel ASSRs, 2) multichannel ASSRs, and 3) single channel EASSRs. A brief description of each model is provided and the model is explicitly defined.

Equations 1 and 2 define the standard discrete state space form all models are placed in [18].

Bold upper case symbols represent matrices, bold lowercase symbols represent vectors and regular lowercase symbols represent numbers. The system state at sample $k$ is represented by $\boldsymbol{x}_{k}$. The state transition model is represented by $\boldsymbol{F}_{k}$ and the process noise is represented by $\boldsymbol{w}_{k}$. The observation model is represented by $\boldsymbol{H}_{k}$ and the measurement noise is represented by $\boldsymbol{v}_{k}$. 


$$
\begin{array}{r}
\boldsymbol{x}_{k+1}=\boldsymbol{F}_{k} \boldsymbol{x}_{k}+\boldsymbol{w}_{k} \\
\boldsymbol{z}_{k}=\boldsymbol{H}_{k} \boldsymbol{x}_{k}+\boldsymbol{v}_{k}
\end{array}
$$

\subsubsection{Acoustic Stimulation Model}

A sinusoid with unknown amplitude and phase is used to model the ASSR [14. This model is presented in equations 3 and 4 . The amplitude at each sample $\left(A_{k}\right)$ is given in equation 5 . The phase at each sample $\left(\phi_{k}\right)$ is given in equation 6 .

$$
\begin{aligned}
& {\left[\begin{array}{l}
x_{1} \\
x_{2}
\end{array}\right]_{k+1}=\left[\begin{array}{ll}
1 & 0 \\
0 & 1
\end{array}\right]\left[\begin{array}{l}
x_{1} \\
x_{2}
\end{array}\right]_{k}+\boldsymbol{w}_{k}}
\end{aligned}
$$

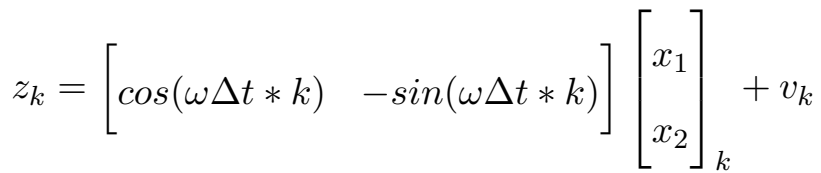

$$
\begin{aligned}
& A_{k}=\sqrt{x_{1, k}^{2}+x_{2, k}^{2}} \\
& \phi_{k}=\arctan \left(\frac{x_{2, k}}{x_{1, k}}\right)
\end{aligned}
$$

\subsubsection{Multichannel Model}

A multi input multi output model was designed to integrate information from multiple channels. The number of channels is represented by $n$. The measurement on channel $i$ and sample $k$ is represented by $z_{i, k}$. This model assumes that each adjacent electrode is measuring the same underlying source with independent noise, this is a common assumption for neighbouring electrodes in high density montages [19]. The same state model in equation 3 is used, but multiple observations are generated as described in equation 7 . 


$$
\left[\begin{array}{c}
z_{1} \\
\vdots \\
z_{n}
\end{array}\right]_{k}=\left[\begin{array}{cc}
\cos (\omega \Delta t * k) & -\sin (\omega \Delta t * k) \\
\vdots & \vdots \\
\cos (\omega \Delta t * k) & -\sin (\omega \Delta t * k)
\end{array}\right]\left[\begin{array}{c}
x_{1} \\
x_{2}
\end{array}\right]_{k}+\boldsymbol{v}_{k}
$$

119

\subsubsection{Electrical Stimulation Model}

Measurements of EASSRs are corrupted by artifacts from RF transmission and electrical stimulation. The exact timing and frequency $f_{a}$ of these artifacts is controlled by the experimenter and thus known a-priori. Figure 1a shows an example EASSR measurement and figure 2a shows the frequency content of this signal. It should be noted that the artifact is $\approx 2000$ times larger than the signal of interest.

A model of the CI artifact is added to the model in section 2.1.1. The artifact is modelled as a linearly increasing and decreasing signal with periodicity equal to the cochlear implant pulse rate $\left(s w\left(\Delta t * k, f_{a}, w\right)\right)$, where $t$ is the width of the artifact. Figure $1 \mathrm{~b}$ shows an example of this artifact model. An additional state variable $\left(x_{3}\right)$ is introduced to track the amplitude of the artifact. The complete EASSR model is provided in equations 8, 9 and 10. An example realisation of the EASSR model overlaid on a measurement can be seen in figure $1 \mathrm{c}$ and the frequency content of this realisation can be seen in figure 2 . 


$$
\begin{gathered}
{\left[\begin{array}{l}
x_{1} \\
x_{2} \\
x_{3}
\end{array}\right]_{k+1}=\left[\begin{array}{lll}
1 & 0 & 0 \\
0 & 1 & 0 \\
0 & 0 & 1
\end{array}\right]\left[\begin{array}{l}
x_{1} \\
x_{2} \\
x_{3}
\end{array}\right]_{k}+w_{k}} \\
\left.z_{k}=\begin{array}{l}
\cos (\omega \Delta t * k)-\sin (\omega \Delta t * k) \\
\left.s w\left(\Delta t * k, f_{a}, w\right)\right] \\
x_{2} \\
x_{3}
\end{array}\right]_{k}+v_{k} \\
\text { sw }\left(\Delta t * k, f_{a}, w\right)=\left\{\begin{array}{l}
1-d / w \quad \text { for }-w \leq d \leq w \\
0
\end{array}\right. \\
\text { where } \mathrm{d}=\min \left(\bmod \left(-t, 1 / f_{a}\right), \bmod \left(t, 1 / f_{a}\right)\right)
\end{gathered}
$$

[Figure 1 about here.]

[Figure 2 about here.]

\subsubsection{Filter}

A standard Kalman filter was applied to the data to determine state estimates at each sample [20]. Initial estimates for $x_{1}$ and $x_{2}$ were set to 0 , representing an amplitude of $0 \mathrm{uV}$. In the EASSR model the initial value of $x_{3}$ was set to the maximum value measured.

The process noise covariance was set to $1 \mathrm{e}^{-7}$ to represent that little variation was expected in response amplitude. The measurement noise covariance was set to the covariance of the measured signal.

A Rauch-Tung-Striebel (RTS) smoother was used in the Kalman filter based analysis [21]. This consists of a forward pass Kalman filter and a backward pass recursion smoother, it provides better estimates than the forward pass alone. 


\section{$2.2 \quad$ Stimuli}

All experiments were conducted in a sound proof and electromagnetically shielded room. EEG measurements were recorded with a BioSemi ActiveTwo 64 channel system. Data was recorded with a sample rate of $8192 \mathrm{~Hz}$ and stored for offline processing. Testing was approved by the Medical Ethics Committee of the UZ Leuven (approval number B32220072126) and informed consent was obtained from all participants.

\subsubsection{Acoustical Data}

Twenty Flemish speakers, mean age years 21 ( $\mathrm{SD}=1.3$ years), volunteered for this study. Stimuli consisted of amplitude modulated speech-weighted noise, presented to the participants at $70 \mathrm{~dB}$ SPL through ER-3A insert phones. Modulation rates 4, 10, 20, 40 and 80 were each presented for 5 minutes in a random order. A resting state condition was also measured for 2.5 minutes. Stimuli were presented binaurally and monaurally to each ear. 16 measurements were taken per participant, in total 320 measurements were recorded. Before further processing the data was downsampled to a sample rate of $256 \mathrm{~Hz}$.

\subsubsection{Electrical Data}

Seven Flemish speakers, mean age 58 years $(\mathrm{SD}=19$ years), volunteered for this study. All participants were unilateral CI users with Cochlear Nucleus devices. Stimuli consisted of amplitude modulated biphasic pulse trains presented via the NIC research platform. All stimuli were presented in a bipolar mode, both the active and return electrodes were located within the cochlea. In each participant three electrode pairs were stimulated, a basal, middle and apical electrode pair. To match the users daily processor, a pulse rate of 900 pulses per second was used, the pulses were modulated at a $40 \mathrm{~Hz}$ rate. Stimuli were modulated between the threshold and comfort level as described in [9]. Each electrode pair was stimulated twice, using either anodic or cathodic first biphasic pulses. In one participant stimulation of the basal electrode pair was not possible. In total 40 measurements were recorded. 


\section{$2.3 \quad$ Signal Processing}

All measurements were processed using the gold standard DFT and proposed Kalman filter methods. Details of each analysis procedure are presented below. Each measurement was analysed to determine the amplitude at the modulation rate. The resting state data was analysed to determine the amplitude at $30 \mathrm{~Hz}$, only noise was expected at this rate.

\subsubsection{DFT Analysis}

The DFT analysis begun with a high pass filter from $2 \mathrm{~Hz}$, each signal was then referenced to electrode Cz. The signal was cut in to epochs of $1.024 \mathrm{~s}, 5 \%$ of these were rejected based on peak to peak amplitude. A Fourier transform was conducted on each epoch and the mean amplitude of the bin corresponding to the modulation rate was recorded as the signal amplitude. The standard deviation of this bin divided by the number of epochs was recorded as the noise amplitude.

\subsubsection{Kalman Analysis}

The Kalman analysis consisted of a de-trending procedure with a second order polynomial over a $0.5 \mathrm{~s}$ window [22]. De-trending was used instead of low pass filtering as not to smear the artifact in the EASSR analysis. The signal was then referenced to $\mathrm{Cz}$ and passed to the Kalman filter and RTS smoother with initial parameters as described in section 2.1.4. The amplitude was estimated at each sample using equation 5, the mean amplitude over all samples was taken for comparison with the DFT analysis.

\subsubsection{Single Channel Analysis}

Single channel analysis was run on electrodes P5, F6, Fp1, and O1. P5 and O1 are commonly used in ASSR analysis as they have a large response amplitude relative to the noise. F6 and Fp1 were analysed to represent more difficult channels for ASSR detection. Fp1 is commonly contaminated with blink artifacts and both Fp1 and F6 are frontal electrodes with a smaller response amplitude than the electrodes at the back of the head. 


\subsubsection{Multi Channel Analysis}

Electrodes P3, P5, PO7 and PO3 were analysed in the multi channel analysis. These are neighbouring electrodes that are commonly used for ASSR analysis. To integrate the information in the traditional processing scheme, the waveforms from multiple channels were averaged after rereferencing to produce a single signal, FFT based analysis was performed on this single channel. In the proposed method, all the channels were passed in to the model as described in equations 3 and 7.

\subsubsection{EASSR Analysis}

The aim of the EASSR analysis was to determine if the evoked response amplitude could be accurately measured in the presence of the CI artifact. The CI artifact is not limited to the stimulation rate, but also contains energy at the modulation rate [8]. The electrode contra-lateral to the CI was analysed, either P5 or P6. As a first step in the DFT analysis the signal was blanked $0.0009 \mathrm{~s}$ after each pulse [8]. This value was chosen as [9] showed that the artifact only lasted 0.0007s with the same stimuli. An anti-aliasing filter was not used prior to interpolation as this smears out the artifact in the time domain, leaving no response dominated samples to interpolate between. For the Kalman analysis equations 8 and 9 was used.

\subsection{Statistics}

Two tests were conducted to ensure the proposed method met or exceeded the performance of the DFT procedure. First, the entire measurements were analysed to confirm the two measures produced the same amplitude estimates, a regression and Bland Altman analysis was conducted. Secondly, the recordings were split in to smaller segments to determine which measure converged to a valid solution quicker.

\subsubsection{Regression Analysis}

To determine if the two methods produce similar amplitude estimates a linear regression was performed comparing the amplitude from a DFT analysis to the Kalman amplitude estimate. The entire 5 min recording was used in both methods. 


\subsubsection{Bland Altman Analysis}

Regression analysis has many shortcomings when comparing two measures [23]. A Bland Altman visual method was used to compare the two measures and define a repeatability coefficient. By definition, $95 \%$ of measurements will have a difference in amplitude estimates less than the repeatability coefficient. This analysis was conducted on the entire data set $(\mathrm{N}=1536)$. As the noise level varies per modulation rate, the Bland Altman analysis was also conducted for each modulation rate. The repeatability coefficient was compared to the noise level at each frequency to determine if the differences between measures was related to the noise level or external factors.

\subsubsection{Convergence Analysis}

Analysis was run to determine which method converged to a valid solution faster. Each measurement was cut in to smaller segments from $10 \mathrm{~s}$ to $250 \mathrm{~s}$ in $10 \mathrm{~s}$ increments. The amplitude as estimated using a DFT analysis of the full 5 minute recording was taken as the true amplitude. The error was defined as the absolute value of the difference between the true amplitude and short segment amplitude. The amplitude estimate was considered valid once the error between it and the 5 min solution was less than the noise level determined on the full 5 min recording using a DFT analysis and remained valid for at least $20 \mathrm{~s}$. The improvement in convergence time was calculated per measurement and for each recording electrode. The mean absolute error was analysed for each segment from $10 \mathrm{~s}$ to $150 \mathrm{~s}$.

\section{Results}

\subsection{Acoustic Stimulation}

\subsubsection{Example}

Figure 3 provides an example of the amplitude estimate over time. Both the DFT and Kalman estimates are visualised. The final DFT estimate is indicated by a dashed black line, the red dashed line indicates the DFT estimate \pm the DFT noise amplitude. The blue line indicates the how the DFT estimate is updated over the measurement. The black line represents the Kalman filter estimate, it is initialised with $0 \mathrm{uV}$ amplitude and converges to within the noise level in $\approx 10 \mathrm{~s}$ 
before fluctuating within the expected noise amplitude range. The green line indicates the RTS smoothed estimate using data from the entire recording.

[Figure 3 about here.]

\subsubsection{Regression Analysis}

Regression analysis showed a significant relation between Kalman and DFT amplitude estimates. Five min ASSR measurements and 2.5 min resting state conditions were analysed. A total of 320 amplitude estimates were obtained with the DFT and Kalman methods per electrode. Four electrodes were analysed to represent both high and low SNR situations. Figure 4 shows the relation between measures for electrode P5. All points lie along the mid line, indicating good agreement. There was a statistically significant relation between measures for all electrodes. For P5 $\left(\mathrm{R}^{2}=0.99\right.$, $p<0.001, \mathrm{DF}=318)$, for $\mathrm{F} 6\left(\mathrm{R}^{2}=0.95, p<0.001, \mathrm{DF}=318\right)$, for Fp1 $\left(\mathrm{R}^{2}=0.92, p<0.001\right.$, $\mathrm{DF}=318)$, for $\mathrm{O} 1\left(\mathrm{R}^{2}=0.99, p<0.001, \mathrm{DF}=318\right)$.

[Figure 4 about here.]

\subsubsection{Bland Altman Analysis}

A Bland Altman analysis indicated the difference in measures is less than the EEG noise. The mean difference between the two measures was $-0.0035 \mathrm{uV}$. For the entire data set the repeatability coefficient was $0.088 \mathrm{uV}$ and the mean noise level was $0.11 \mathrm{uV}$.

Figure 5 shows a Bland Altman plot. The repeatability coefficient is indicated by dashed lines. The average noise level is plotted as a dotted line.

Figure 6 plots the average noise per electrode and frequency against the repeatability coefficient. The points generally lie above the solid line indicating the difference in measures is less than the frequency specific measurement noise.

[Figure 5 about here.]

[Figure 6 about here.] 


\subsubsection{Multichannel Analysis}

Regression analysis showed that the Kalman and DFT analysis estimated the amplitude similarly when integrating information from multiple channels. DFT analysis averaged the signals in time domain then the standard processing was used. For the Kalman process all channels were fed to the filter. Regression analysis determined the two measures were related $\left(\mathrm{R}^{2}=0.99, p<0.001\right.$, $\mathrm{DF}=318)$. The Bland Altman repeatability coefficient was $0.048 \mathrm{uV}$. The mean noise level was $0.075 \mathrm{uV}$. Figure 7 illustrates the similarity between DFT and Kalman estimated amplitudes for the multi channel data.

[Figure 7 about here.]

\subsubsection{Time Analysis}

Figure 8 illustrates how the mean absolute amplitude error varies with the length of recording for two electrodes and two modulation rates, the dashed horizontal line indicates the average noise level. Results are described for each frequency as the noise level decreases with increasing frequency. For each modulation rate the amplitude was considered valid when the mean absolute error was less than the mean noise level as determined on the full 5 min recording.

The improvement in convergence time was calculated for each measurement on each of the four recording electrodes. On average the proposed Kalman approach converged to a solution $15 \%$ faster than the DFT method, a t test indicates this is a significant improvement $\mathrm{t}(1279)=5.7, \mathrm{p}<$ .001. For $4 \mathrm{~Hz}$ conditions the improvement was $27 \% \mathrm{t}(239)=6.0, \mathrm{p}<.001$. For $10 \mathrm{~Hz}$ conditions the improvement was $26 \% \mathrm{t}(239)=4.8, \mathrm{p}<.001$. For $20 \mathrm{~Hz}$ conditions the improvement was 3 $\% \mathrm{t}(239)=0.2, \mathrm{p}=.5$. For $40 \mathrm{~Hz}$ conditions the improvement was $10 \% \mathrm{t}(239)=1.5, \mathrm{p}=.1$. For $80 \mathrm{~Hz}$ conditions the improvement was $6 \% \mathrm{t}(239)=0.8, \mathrm{p}=.4$. For resting state data the improvement was $27 \% \mathrm{t}(79)=4.0, \mathrm{p}<.05$.

When all conditions and modulation rates were combined, the mean absolute error for the Kalman filter achieved a valid amplitude on average between 50 and $60 \mathrm{~s}$, the traditional method between 80 and $90 \mathrm{~s}$. For $4 \mathrm{~Hz}$ the Kalman filter achieved a valid amplitude on average between 50 and $60 \mathrm{~s}$, the traditional method between 80 and $90 \mathrm{~s}$. For $10 \mathrm{~Hz}$ the Kalman filter achieved a valid amplitude on average between 60 and $70 \mathrm{~s}$, the traditional method between 90 and $100 \mathrm{~s}$. For 
$20 \mathrm{~Hz}$ the Kalman filter achieved a valid amplitude on average between 80 and $90 \mathrm{~s}$, the traditional method between 80 and $90 \mathrm{~s}$. For $40 \mathrm{~Hz}$ the Kalman filter achieved a valid amplitude on average between 90 and $100 \mathrm{~s}$, the traditional method between 100 and $110 \mathrm{~s}$. For $80 \mathrm{~Hz}$ the Kalman filter achieved a valid amplitude on average between 30 and $40 \mathrm{~s}$, the traditional method between 70 and 80 s. For resting state data the Kalman filter achieved a valid amplitude on average between 10 and $20 \mathrm{~s}$, the traditional method between 50 and $60 \mathrm{~s}$.

[Figure 8 about here.]

\subsection{Electrical Stimulation}

The proposed method determined the EASSR amplitude to be the same as the DFT based blanking method for all participants. Artifact amplitudes varied between participants from $5 \mathrm{uV}$ to $2000 \mathrm{uV}$. The width of the artifact was chosen by visual inspection of the raw EEG data.

\subsubsection{Regression Analysis}

Regression analysis confirmed the amplitudes from the two methods are related $\left(R^{2}=0.94, p<\right.$ $0.001, \mathrm{df}=38)$

\subsubsection{Bland Altman Analysis}

Figure 9 shows the Bland Altman analysis for the EASSR files. The Bland Altman analysis determined the mean difference between methods to be $0.006 \mathrm{uV}$. The repeatability coefficient was 0.047 $\mathrm{uV}$, the repeatability coefficient for acoustic ASSRs at $40 \mathrm{~Hz}$ was $0.051 \mathrm{uV}$.

[Figure 9 about here.]

\section{Discussion}

A method for the real time analysis of ASSR signals was presented. For recordings of 5 and 2.5 min, the proposed method produced the same amplitude estimates as the gold standard DFT method. The variation in measures was shown to be less than the EEG noise level. The proposed Kalman approach reached a valid amplitude significantly faster than the DFT approach. Two extensions of 
this method were presented. The first, integrated information from multiple sensors. The second, allowed measurement of responses to CI stimulation.

For all modulation rates, the 5 min recordings produced on average essentially no difference in amplitude when compared to the DFT method. This indicates no bias is introduced in the proposed method, and is an essential requirement for any new measure. The variation in results was shown to be less than the EEG noise level. The authors propose this indicates the two measures can be considered equally accurate for long recordings. The two measures produced the same amplitude estimate for the resting state condition, indicating the Kalman filter approach also works in the absence of a significant neural response to the stimuli. Differences between the two methods may be due to variation or non stationarity in the ASSR amplitude over the length of the recording, this variation in the frequency of interest is not usually discussed in DFT based methods.

The recordings were analysed at different lengths to determine which converged to a valid solution first. A valid solution was defined as when the absolute error in amplitude was less than the noise level for the full recording for at least $20 \mathrm{~s}$. For all modulation rates the Kalman filter matched or outperformed the DFT method, the Kalman filter was significantly faster for three modulation rates. The greatest improvement in convergence time was found in low SNR situations such as frontal electrodes and low modulation rates. The authors propose two reasons for the improvement in detection time. First, the initial condition is set to an amplitude of $0 \mathrm{uV}$ and increases based on the measurements, whereas DFT based methods often over estimate response amplitudes for short measurements. Secondly, the Kalman filter is less likely to be affected by brief periods of noisy data caused by blinking or neck movement. Given the greater reduction in measurement time for frontal electrodes, the authors hypothesise that the proposed method may further outperform DFT methods for stimuli presented closer to hearing threshold.

The proposed Kalman filter was extended to integrate information from multiple electrodes. The model is based on the assumption that each electrode is measuring the same underlying signal but with independent noise, this assumption is not strictly true [24, 25. However, closely spaced electrodes often show similar amplitude and phase and are combined [11]. For entire scalp analysis, other methods may be more appropriate [13. Less variability was shown when comparing amplitudes with the DFT method for the multiple electrode data than the single channel data. Indicating a benefit of integrating information from multiple sensors. 
A model was developed to include the artifacts introduced when recording EEG with CI stimulation. The artifacts are caused by RF transmission and electrical stimulation. The artifacts from participants in this study varied in amplitude between $5 \mathrm{uV}$ and $2000 \mathrm{uV}$. A simple linearly increasing and decreasing model was proposed, this was based on visual inspection of raw EEG data and the signal spectrum. Realisations of the model showed good visual alignment with raw data. Kalman filtering of EASSR data with the extended model showed no difference in amplitudes compared to the current state of the art artifact removal method [8]. If the artifact model was not included in the Kalman filter, the amplitude estimates were inaccurate. The repeatability coefficient between methods for the $40 \mathrm{~Hz}$ EASSR was similar to the $40 \mathrm{~Hz}$ ASSR, indicating no loss of accuracy is produced relative to measurements in normal hearing participants. The proposed method has the same advantages when processing EASSR data as with the ASSR data, but also has the advantage that it does not require interpolation, which effectively downsamples without pre-filtering, causing aliasing in the signal.

CI stimuli were presented at 900 pulses per second in bipolar mode, these parameters have been previously validated with the blanking technique [8]. The CI artifact is known to vary with stimulation mode and level, and may vary between CI manufacturers. Further investigation is required to determine how the artifact varies with each stimulation parameter. If stimulation parameters dramatically affect the measured artifact, the model in equation 10 may need to be adjusted accordingly. However, the participants in this study had a representative range of threshold and comfort values, this demonstrated that the Kalman filter was robust to variations in stimulation level.

The DFT analysis methods assume the neural response is stationary, this may not be true as attention and alertness are known to affect auditory neural responses [10]. For the standard Kalman filter to provide an optimal estimate of state variables the model must be correct and noise must be white Gaussian. Figure 2a illustrates that the EEG signal does not have white noise. Despite this limitation the Kalman filter outperforms the DFT based method and does not require the stationarity assumption. Using methods that account for correlated noise may provide further improvements [26].

Initial conditions were set to an amplitude of $0 \mathrm{uV}$ as not to bias the comparison to DFT based methods. In an optimised system, the initial estimate could be set per modulation rate to 
the expected amplitude based on literature or pilot studies, this should further reduce the time required to converge to a valid amplitude estimate. Further, tracking the response harmonics could be included by simple extension of the model [27].

An alternative approach to validating the proposed Kalman filter, and comparing to the DFT approach, could be to simulate a neural response in noise and compare the results to the simulation parameters. This would allow analysis of the error in different conditions, but would lack the complexity of the experimental data.

\section{Conclusion}

A new method was proposed to measure auditory steady state responses. The proposed Kalman filter method produced the same amplitude estimates as the gold standard DFT based method for long measurements. The proposed method was shown to converge to a valid solution significantly faster than the current gold standard. The proposed method was extended to include a model of cochlear implant artifacts, and was shown to accurately estimate the amplitude of electrically evoked auditory steady state responses. The authors hope that removing the assumption of stationarity and allowing real time amplitude estimation will open up new avenues in ASSR research. 


\section{Appendix}

\section{Acknowledgment}

The work leading to this deliverable and the results described therein has received funding from the People Programme (Marie Curie Actions) of the European Unions Seventh Framework Programme FP7/2007-2013/ under REA grant agreement PITN-GA-2012-317521.

This study was performed as part of the Agency for Innovation by Science and Technology project number 150432 in cooperation with Cochlear Ltd.

The authors thank Elisabeth Van Eldere and Marlies Buys for their assistance in collecting the EEG data. We thank the reviewers and editor for their constructive input. 


\section{References}

[1] T. W. Picton, Human Auditory Evoked Potentials. San Diego: Plural, 2010.

[2] G. Rance, F. W. Rickards, L. T. Cohen, S. De Vidi, and G. M. Clark, "The automated prediction of hearing thresholds in sleeping subjects using auditory steady-state evoked potentials," Ear and Hearing, vol. 16, no. 5, pp. 499-507, 1995.

[3] H. Luts, C. Desloovere, A. Kumar, E. Vandermeersch, and J. Wouters, "Objective assessment of frequency-specific hearing thresholds in babies," International journal of pediatric otorhinolaryngology, vol. 68, no. 7, pp. 915-926, 2004.

[4] A. Dimitrijevic, M. S. John, and T. W. Picton, "Auditory steady-state responses and word recognition scores in normal-hearing and hearing-impaired adults," Ear and Hearing, vol. 25, no. 1, pp. 68-84, 2004.

[5] H. Poelmans, H. Luts, M. Vandermosten, B. Boets, P. Ghesquière, and J. Wouters, "Auditory steady state cortical responses indicate deviant phonemic-rate processing in adults with dyslexia," Ear and Hearing, vol. 33, no. 1, pp. 134-143, 2012.

[6] T. W. Picton, J. Vajsar, R. Rodriguez, and K. B. Campbell, "Reliability estimates for steadystate evoked potentials," Electroencephalography and Clinical Neurophysiology/Evoked Potentials Section, vol. 68, no. 2, pp. 119-131, 1987.

[7] M. Hofmann and J. Wouters, "Electrically evoked auditory steady state responses in cochlear implant users," Journal of the Association for Research in Otolaryngology, vol. 11, no. 2, pp. 267-82, Jun 2010.

[8] — _ "Improved electrically evoked auditory steady-state response thresholds in humans," Journal of the Association for Research in Otolaryngology, vol. 13, no. 4, pp. 573-589, 2012.

[9] R. Luke, L. Van Deun, M. Hofmann, A. Van Wieringen, and J. Wouters, "Assessing temporal modulation sensitivity using electrically evoked auditory steady state responses," Hearing Research, vol. 324, pp. 37-45, 2015. 
[10] S. I. Kanoh, K.-i. Miyamoto, and T. Yoshinobu, "A brain-computer interface (bci) system based on auditory stream segregation," in Engineering in Medicine and Biology Society, 2008. EMBS 2008. 30th Annual International Conference of the IEEE. IEEE, 2008, pp. 642-645.

[11] S. Vanvooren, H. Poelmans, M. Hofmann, P. Ghesquière, and J. Wouters, "Hemispheric asymmetry in auditory processing of speech envelope modulations in prereading children," The Journal of Neuroscience, vol. 34, no. 4, pp. 1523-1529, 2014.

[12] A. De Cheveigné and J. Z. Simon, "Denoising based on spatial filtering," Journal of Neuroscience Methods, vol. 171, no. 2, pp. 331-339, 2008.

[13] H. M. Bharadwaj and B. G. Shinn-Cunningham, "Rapid acquisition of auditory subcortical steady state responses using multichannel recordings," Clinical Neurophysiology, vol. 125, no. 9, pp. 1878-1888, 2014.

[14] A. A. Girgis and R. G. Brown, "Application of kalman filtering in computer relaying," Power Apparatus and Systems, IEEE Transactions on, no. 7, pp. 3387-3397, 1981.

[15] B. Oken, M. Salinsky, and S. Elsas, "Vigilance, alertness, or sustained attention: physiological basis and measurement," Clinical Neurophysiology, vol. 117, no. 9, pp. 1885-1901, 2006.

[16] R. R. Bitmead, A. C. Tsoi, and P. J. Parker, "A kalman filtering approach to short-time fourier analysis," Acoustics, Speech and Signal Processing, IEEE Transactions on, vol. 34, no. 6, pp. 1493-1501, 1986.

[17] X. Li, Y. Sokolov, and H. Kunov, "System and method for processing low signal-to-noise ratio signals," Oct. 23 2007, uS Patent 7,286,983.

[18] B. D. Anderson and J. B. Moore, Optimal Filtering. Courier Corporation, 2005.

[19] A. De Cheveigné and J. Z. Simon, "Sensor noise suppression," Journal of Neuroscience Methods, vol. 168, no. 1, pp. 195-202, 2008.

[20] B. Anderson and J. B. Moore, "Optimal filtering," Prentice-Hall Information and System Sciences Series, Englewood Cliffs: Prentice-Hall, 1979, vol. 1, 1979. 
[21] H. E. Rauch, C. Striebel, and F. Tung, "Maximum likelihood estimates of linear dynamic systems," AIAA journal, vol. 3, no. 8, pp. 1445-1450, 1965.

[22] A. Savitzky and M. J. Golay, "Smoothing and differentiation of data by simplified least squares procedures." Analytical chemistry, vol. 36, no. 8, pp. 1627-1639, 1964.

[23] J. M. Bland and D. Altman, "Statistical methods for assessing agreement between two methods of clinical measurement," The lancet, vol. 327, no. 8476, pp. 307-310, 1986.

[24] B. Roß, C. Borgmann, R. Draganova, L. E. Roberts, and C. Pantev, "A high-precision magnetoencephalographic study of human auditory steady-state responses to amplitude-modulated tones," The Journal of the Acoustical Society of America, vol. 108, no. 2, pp. 679-691, 2000.

[25] P. L. Nunez, R. Srinivasan, A. F. Westdorp, R. S. Wijesinghe, D. M. Tucker, R. B. Silberstein, and P. J. Cadusch, "Eeg coherency: I: statistics, reference electrode, volume conduction, laplacians, cortical imaging, and interpretation at multiple scales," Electroencephalography and clinical neurophysiology, vol. 103, no. 5, pp. 499-515, 1997.

[26] K. Wang, Y. Li, and C. Rizos, "Practical approaches to kalman filtering with time-correlated measurement errors," Aerospace and Electronic Systems, IEEE Transactions on, vol. 48, no. 2, pp. 1669-1681, 2012.

[27] N. C. Will and R. Cardoso, "Comparative analysis between fft and kalman filter approaches for harmonic components detection," in Industry Applications (INDUSCON), 2012 10th IEEE/IAS International Conference on. IEEE, 2012, pp. 1-7. 
477

478

479

480

481

482

483

484

485

486

487

488

489

490

491

492

493

494

495

496

497

498

500

501

\section{List of Figures}

\begin{tabular}{|c|c|}
\hline 1 & Example EASSR measurement and model realisation. 1a) EASSR measurement, |1b) \\
\hline & realisation of artifact model sampled at $81920 \mathrm{~Hz}$ to capture all features, 1 1c) EASSR \\
\hline & model realisation (red) overlaid on example measurement (black). . \\
\hline 2 & Spectrum of EASSR measurement and model realisation. \\
\hline 3 & Example amplitude estimation for a $4 \mathrm{~Hz}$ modulation rate using both Kalman and \\
\hline & DFT measures. Time varying estimates are shown as solid lines. The dashed black \\
\hline & line indicates the DFT amplitude estimate using the entire 5 min measurement, the \\
\hline & dashed red line indicates the amplitude estimate \pm the final noise amplitude. \\
\hline 4 & Comparison of amplitude estimates for electrode P5 using DFT and Kalman methods. \\
\hline & Solid line indicates the line of perfect match. \\
\hline 5 & Bland Altman analysis of difference between DFT and Kalman amplitude estimates \\
\hline & for single channel analysis of six modulation rates and 4 electrodes $(\mathrm{N}$ \\
\hline & dicate the repeatability coefficient and dotted lines indicate the mean \\
\hline & DFT noise level. The rug indicates the density of distribution for each axis. \\
\hline 6 & Relation between repeatability coefficient and noise. \\
\hline 7 & Bland Altman analysis of difference between DFT and Kalman amplitude estimates \\
\hline & "320). Dashed lines indicate the repeatability coefficient \\
\hline & and dotted lines indicate the mean DFT noise level. Tl \\
\hline & distribution for each axis. \\
\hline 8 & Error in amplitude estimates as a function of time for two electrodes and two modu- \\
\hline & lation rates. Error bars indicate $95 \%$ confidence intervals. Dashed lines indicate the \\
\hline & noise amplitude. \\
\hline 9 & Bland Altman analysis for EASSR recordings. Dashed lines indicate the \\
\hline & bility coefficient. The rug indicates the de \\
\hline & h axis. \\
\hline
\end{tabular}




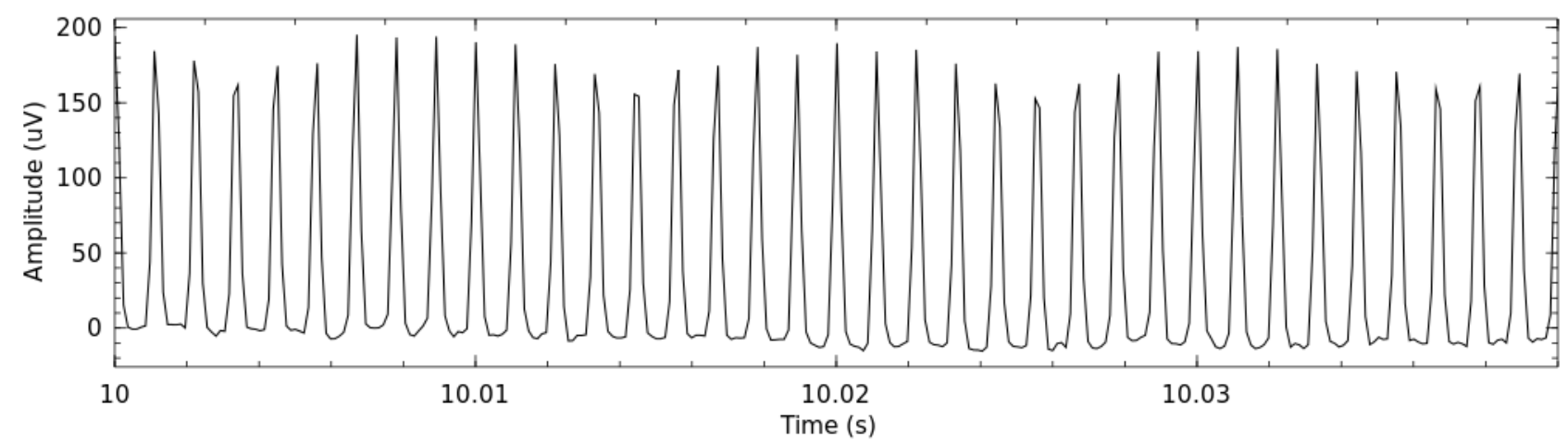

(a) EASSR Signal sampled at $8192 \mathrm{~Hz}$

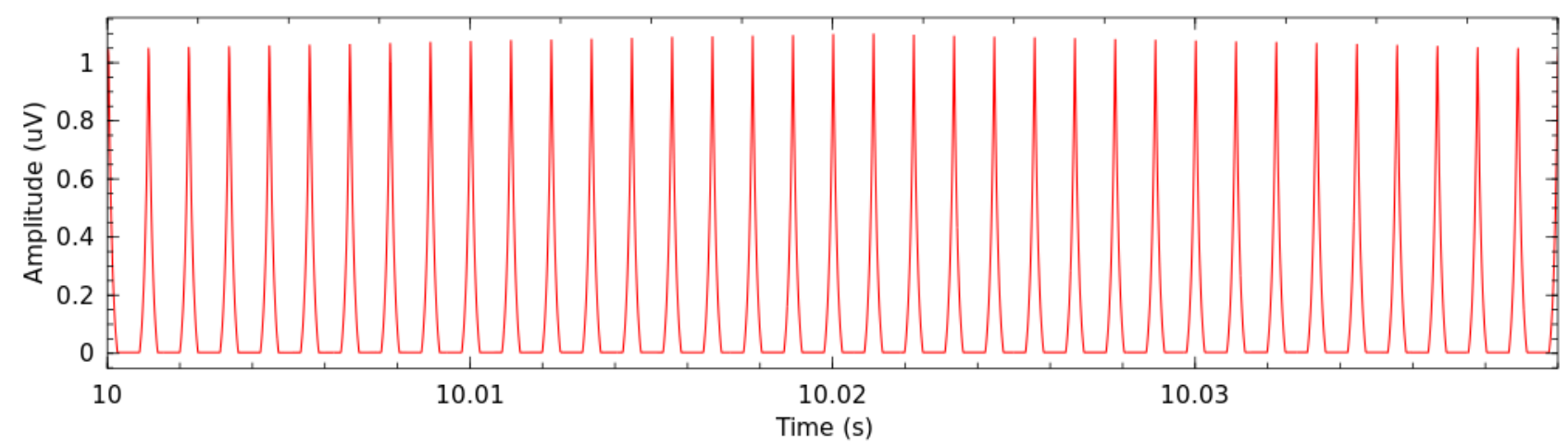

(b) Artifact Model sampled at $81920 \mathrm{~Hz}$

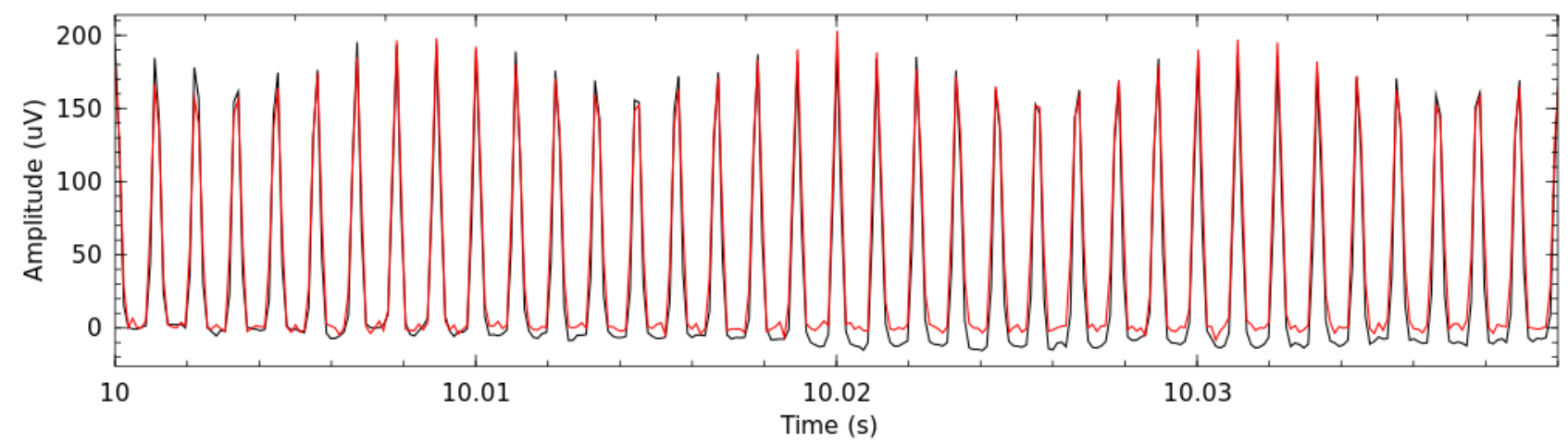

(c) Measurement and model realisation

Figure 1: Example EASSR measurement and model realisation. 1a EASSR measurement, 1b) realisation of artifact model sampled at $81920 \mathrm{~Hz}$ to capture all features, 1c EASSR model realisation (red) overlaid on example measurement (black). 


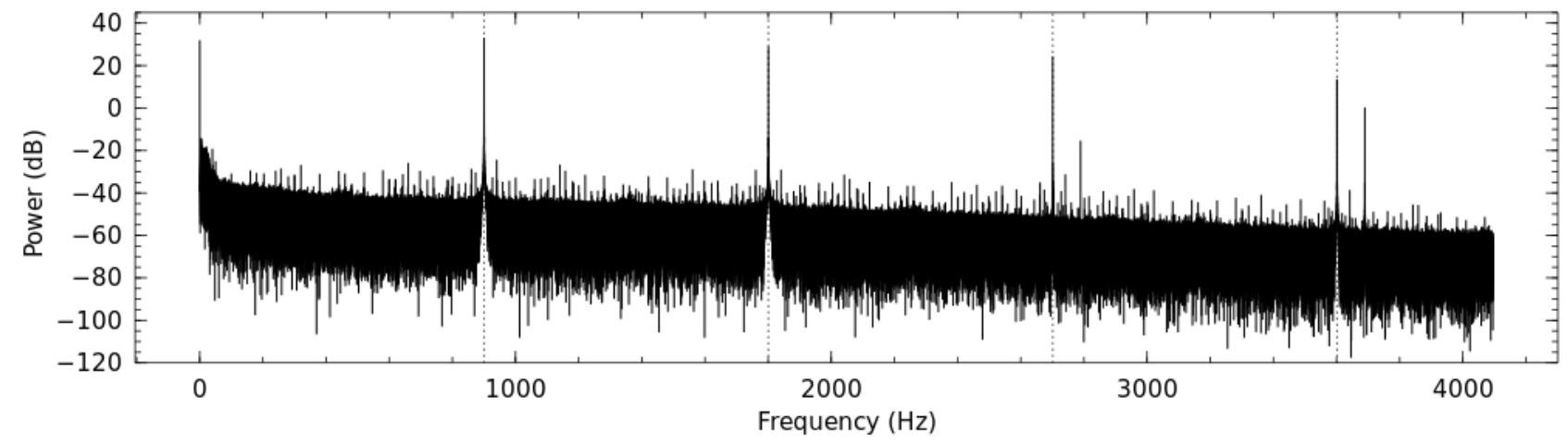

(a) Measurement

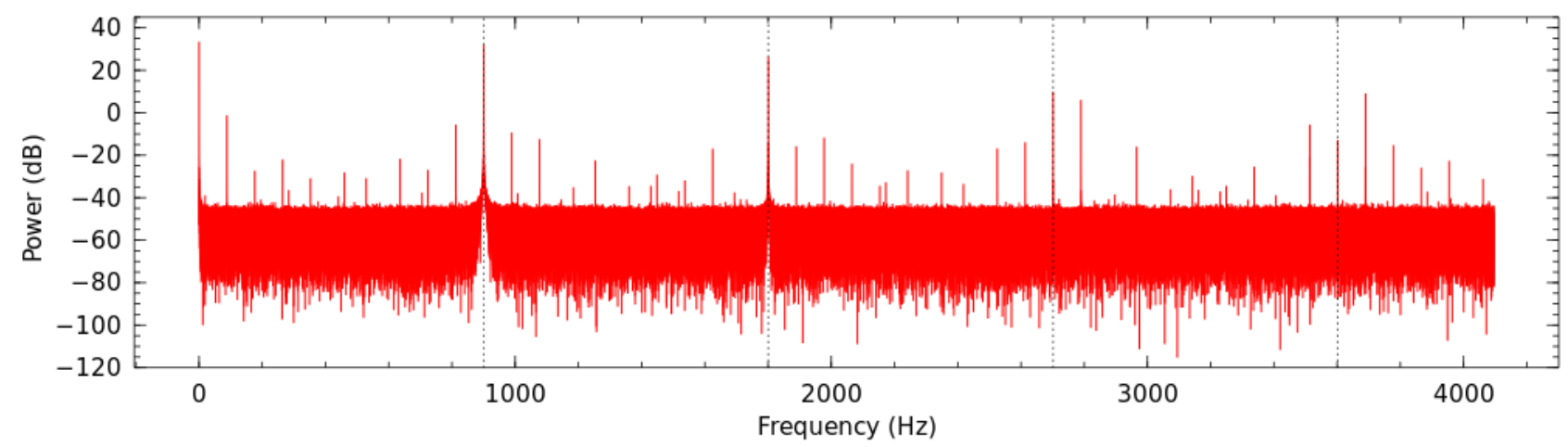

(b) Model

Figure 2: Spectrum of EASSR measurement and model realisation. 


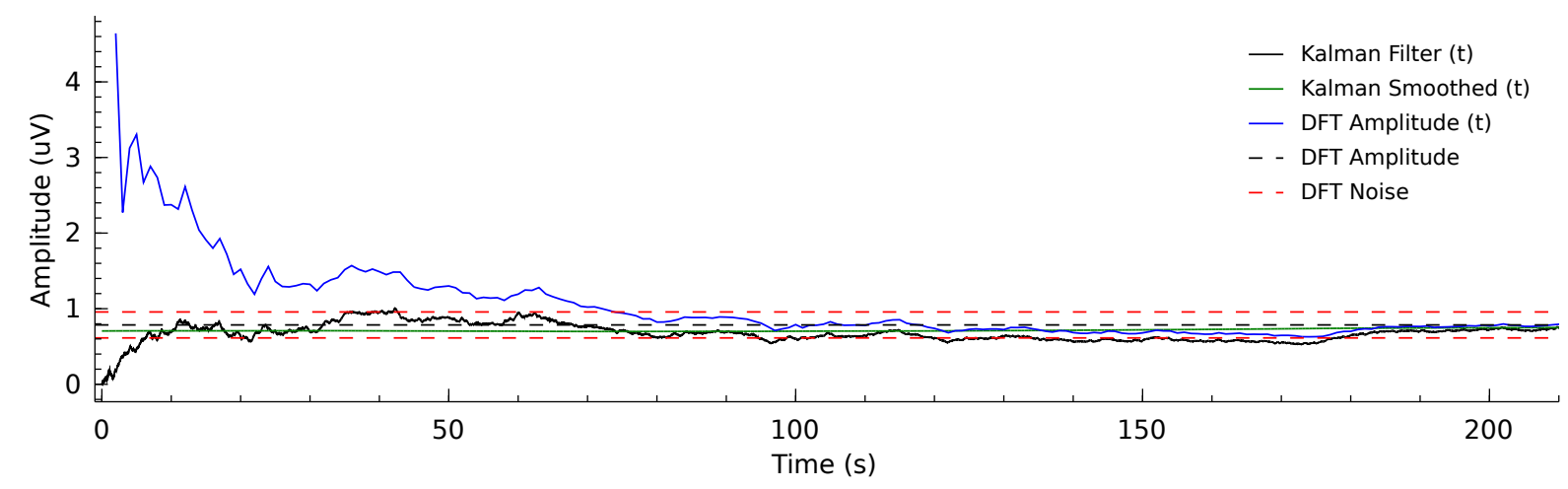

Figure 3: Example amplitude estimation for a $4 \mathrm{~Hz}$ modulation rate using both Kalman and DFT measures. Time varying estimates are shown as solid lines. The dashed black line indicates the DFT amplitude estimate using the entire 5 min measurement, the dashed red line indicates the amplitude estimate \pm the final noise amplitude. 


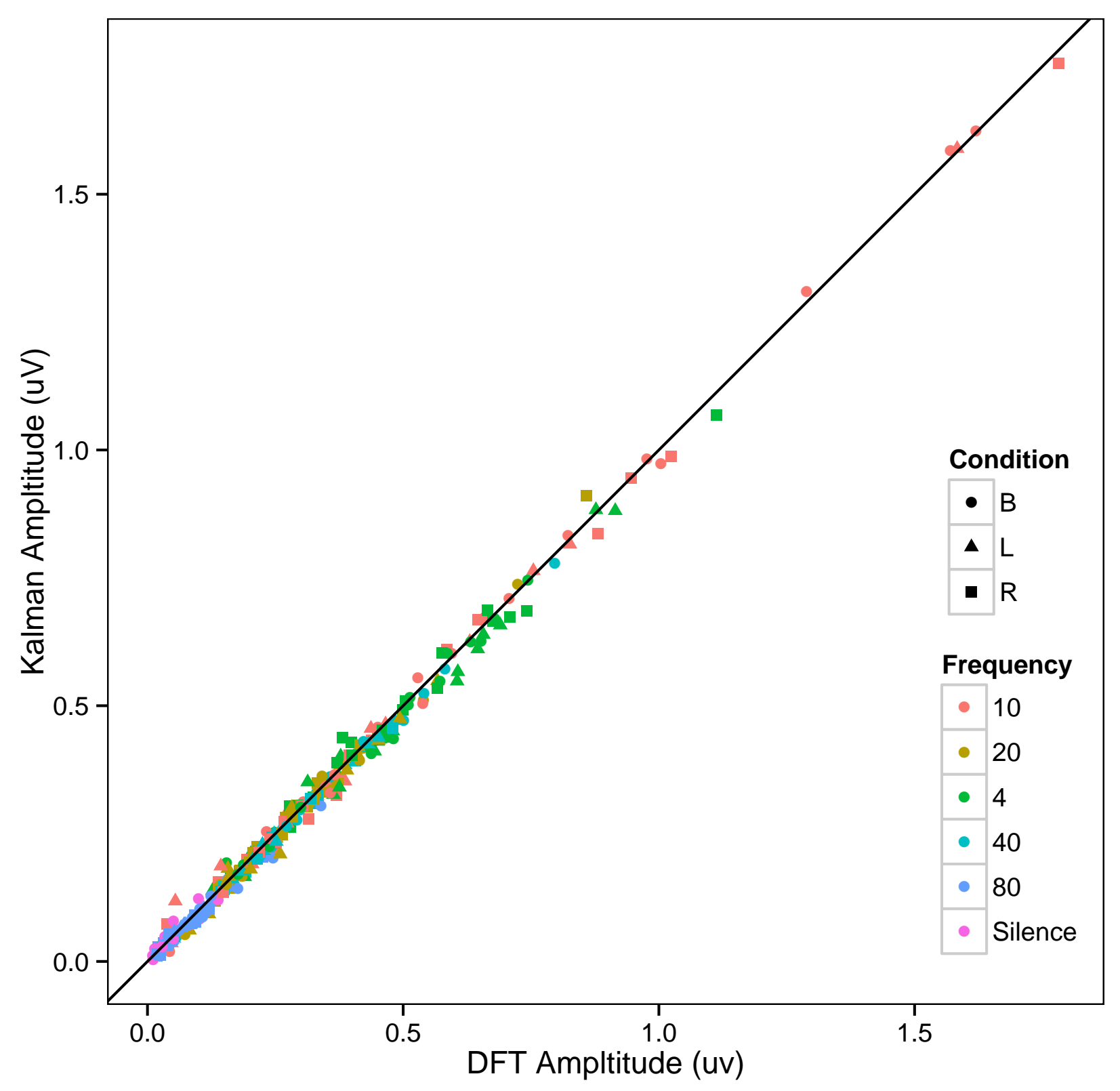

Figure 4: Comparison of amplitude estimates for electrode P5 using DFT and Kalman methods. Solid line indicates the line of perfect match. 


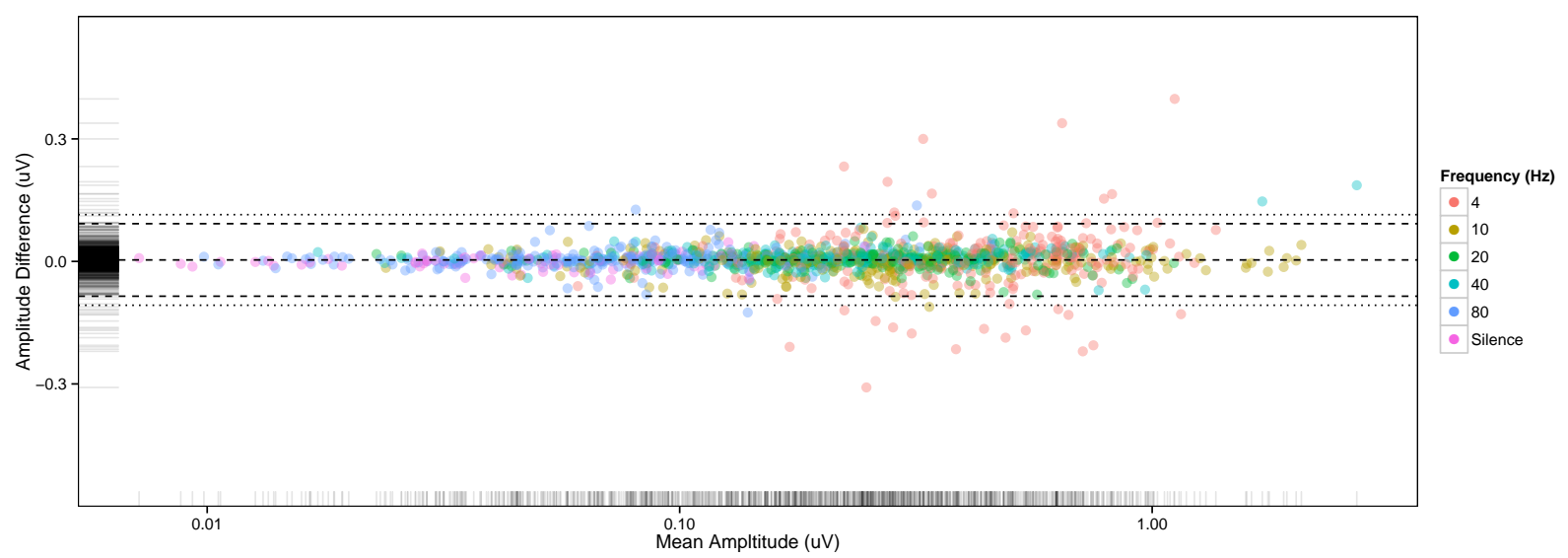

Figure 5: Bland Altman analysis of difference between DFT and Kalman amplitude estimates for single channel analysis of six modulation rates and 4 electrodes $(\mathrm{N}=1,280)$. Dashed lines indicate the repeatability coefficient and dotted lines indicate the mean DFT noise level. The rug indicates the density of distribution for each axis. 


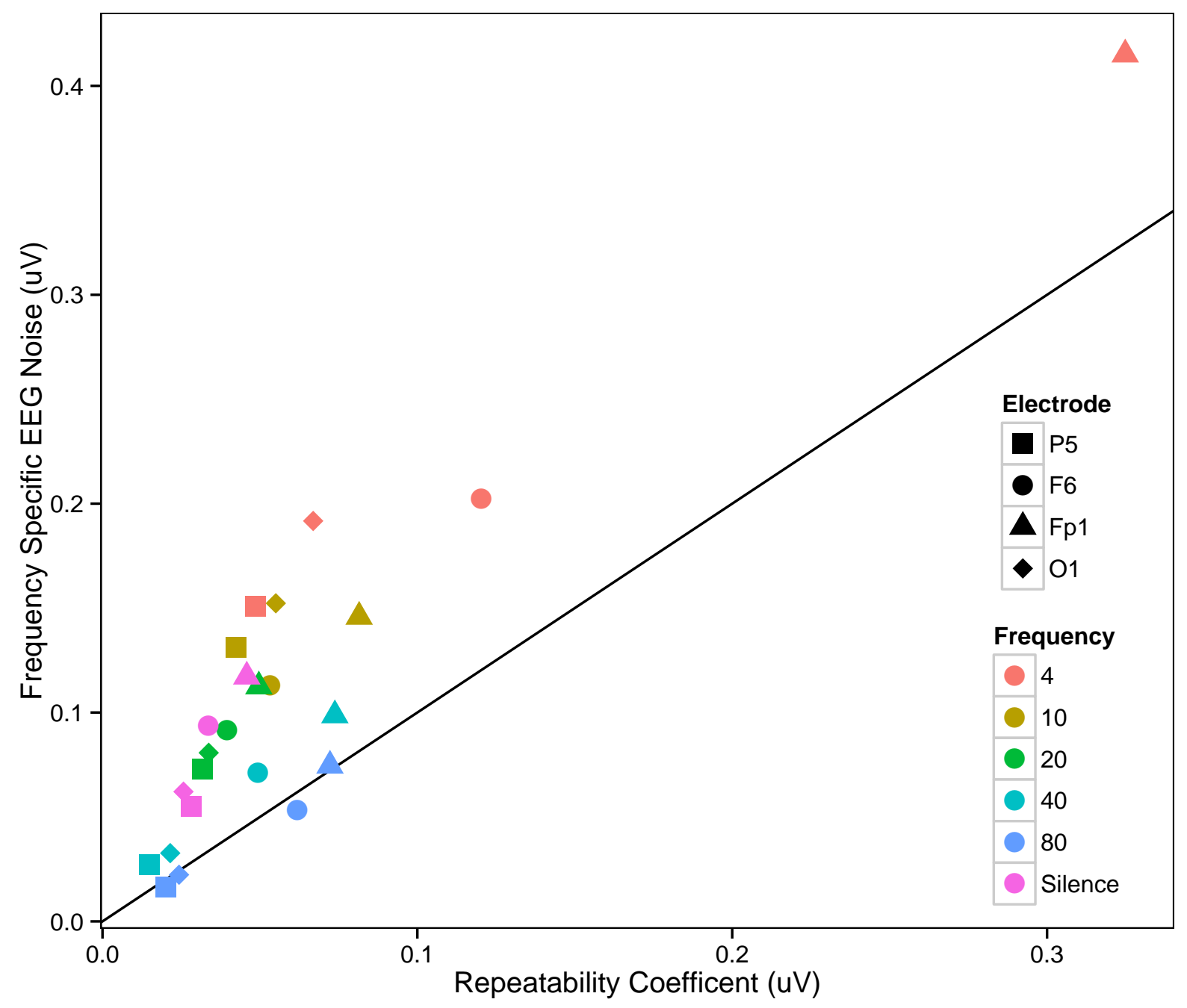

Figure 6: Relation between repeatability coefficient and noise. 


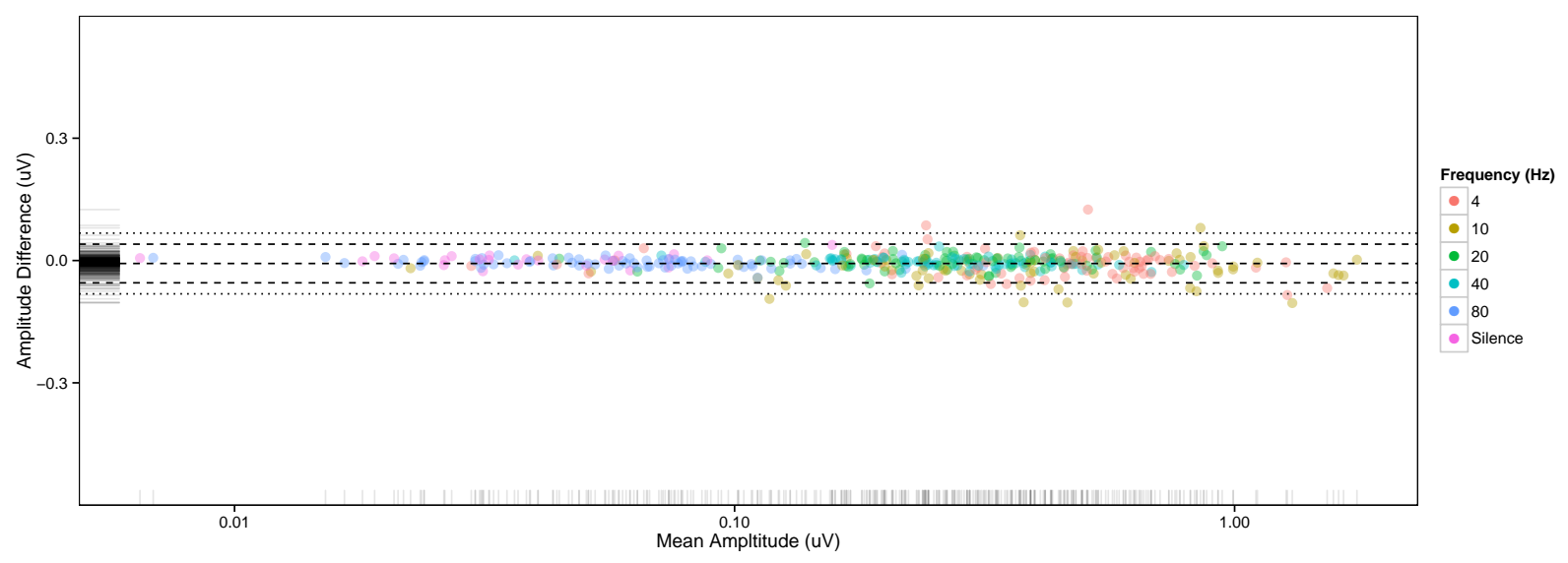

Figure 7: Bland Altman analysis of difference between DFT and Kalman amplitude estimates for multi channel data $(\mathrm{N}=320)$. Dashed lines indicate the repeatability coefficient and dotted lines indicate the mean DFT noise level. The rug indicates the density of distribution for each axis. 


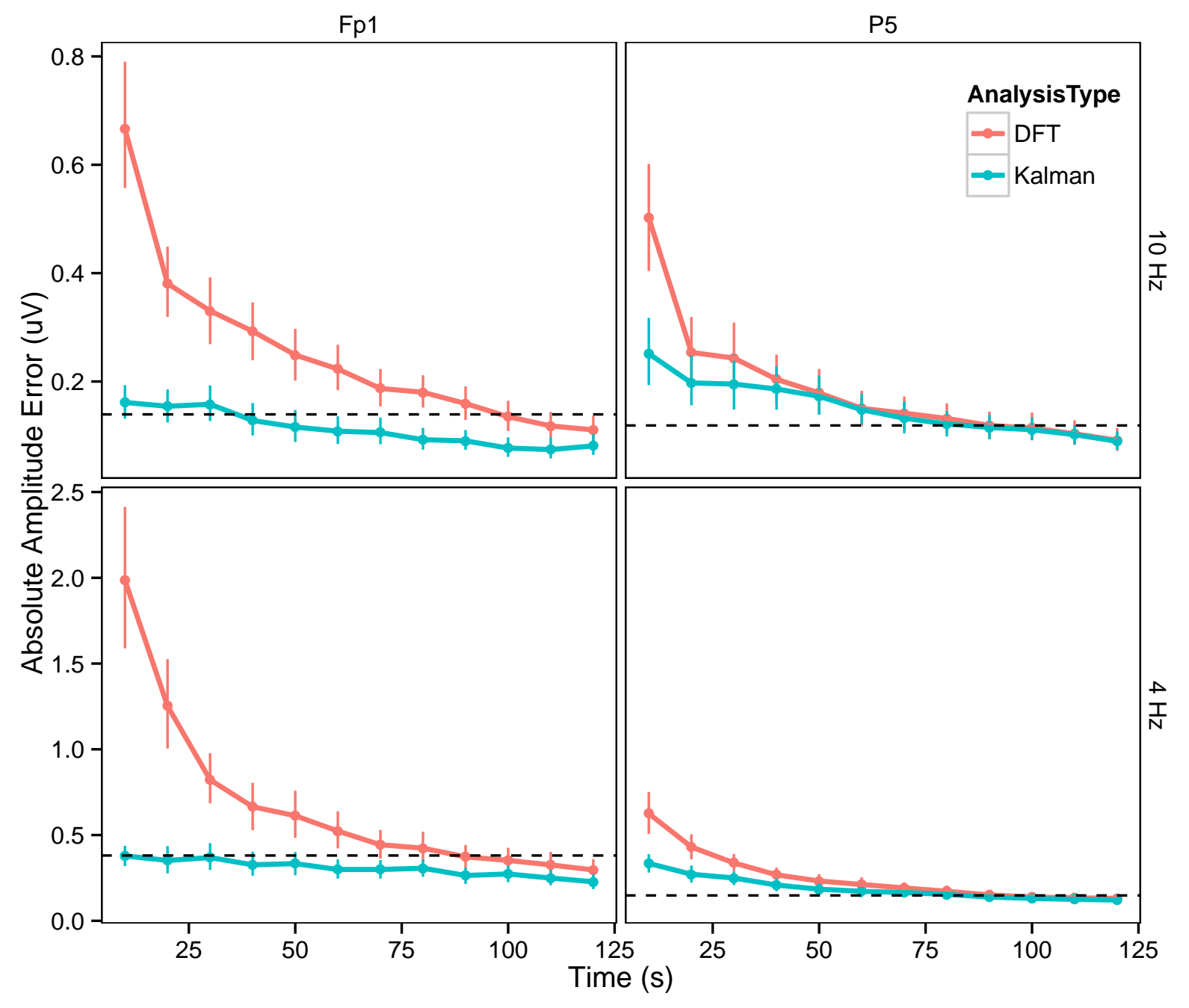

Figure 8: Error in amplitude estimates as a function of time for two electrodes and two modulation rates. Error bars indicate $95 \%$ confidence intervals. Dashed lines indicate the noise amplitude. 


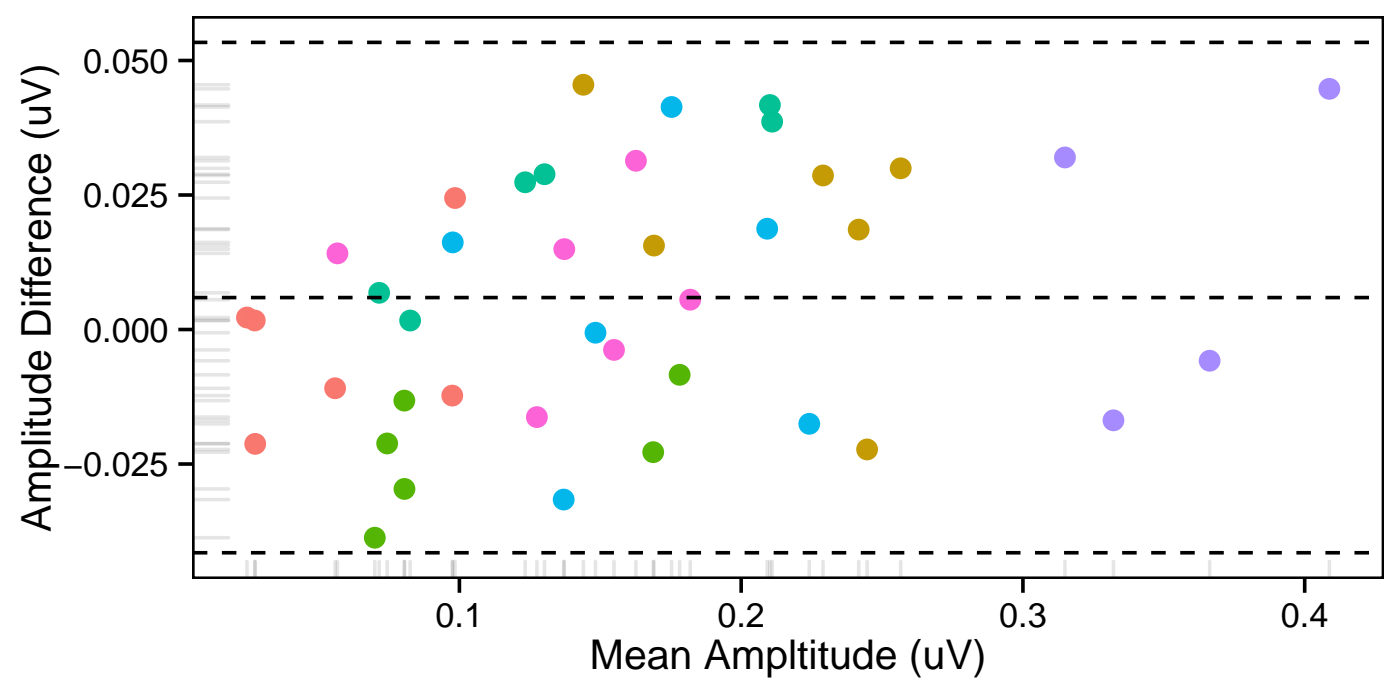

Participant

- P1

- P2

- P3

- P4

- P5

- P6

- P7

Figure 9: Bland Altman analysis for EASSR recordings. Dashed lines indicate the mean difference and repeatability coefficient. The rug indicates the density of distribution for each axis. 